\title{
Epidemiología de la fractura de cadera en ancianos en España
}

\author{
J. A. SERRA, G. GARRIDO*, M. VIDÁN, E. MARAÑÓN, F. BRAÑAS, J. ORTIZ \\ Servicio de Geriatría. Hospital General Universitario Gregorio Marañón. \\ *Organización Nacional de Trasplantes. Madrid
}

\author{
EPIDEMIOLOGY OF HIP FRACTURE IN ELDERLY IN SPAIN
}

\section{RESUMEN}

Fundamento: El 90\% de las fracturas de cadera (FC) ocurren en mayores de 64 años. Pretendemos describir las características epidemiológicas de los ancianos con FC en lo que respecta a edad, sexo, época de ingreso, estancia hospitalaria y mortalidad en las distintas Comunidades Autónomas (CCAA) de España.

Método: Se analizan datos de incidencia de FC (Código 820.0 al 820.9 de la Clasificación Internacional de Enfermedades) entre los años 1996 y 1999, obtenidos del registro nacional del Conjunto Mínimo Básico de Datos del Ministerio de Sanidad. Los datos de la población se obtienen del Instituto Nacional de Estadística. Las tasas de FC se calculan mediante densidad de incidencia bruta y ajustada. Se analizan los factores que influyen en la mortalidad hospitalaria mediante análisis multivariante.

Resultados: De las 146.383 FC estudiadas 130.414 (89\%) corresponden a mayores de 64 años, con una edad media de 82 años (78\% mujeres). Existe una gran variabilidad en los resultados entre las CCAA. La estancia media hospitalaria es de 16 días y la mortalidad hospitalaria del $5 \%$. La incidencia global de FC es de 517 casos por 100.000 ancianos y año (270 casos/100.000 varones y 695/100.000 mujeres). La mortalidad hospitalaria es el doble en varones que en mujeres $(8,1 \%$ y $4,2 \%$ respectivamente), aumenta exponencialmente con la edad, y es mayor en invierno. Los más ancianos, varones, que se fracturan en invierno y que no habitan en las Comunidades del Mediterráneo y Canarias tienen más probabilidades de fallecer en el hospital tras una FC.

Conclusiones: La FC es una patología afecta preferentemente a los ancianos, más frecuentemente en mujeres y con una gran variación en la incidencia, mortalidad y estancia hospitalaria entre las distintas CCAA.

PALABRAS CLAVE: Fractura de cadera. Anciano. Epidemiología.

\section{ABSTRACT}

Background: Ninety percent of hip fractures $(H F)$ occur in people older than 64 years. We describe the epidemiological data (age, sex, date of admission and discharge and mortality) of elderly with hip fracture in the different regions of Spain.

Method: Data obtained from the Minimum Data Set of the Ministry of Health were used to analyse hip fracture incidence (Identified by codes 820.0 through 820.9 of the International Classification of Dise ases) from 1996 through 1999. Demographic data of Spanish population were obtained from the National Institute of Statistics. HF rates were calculated using crude and age-and-sex adjusted density of incidence. Factors associated with in-hospital mortality were assessed by multiva riate analyses.

Results: $89 \%$ (130.414) of 146.383 HF cases studied were in elderly patients with a mean age of 82 years old, being $78 \%$ females. The results showed a great variability through the different regions. Length of hospi tal stay was 16 days, and in-hospital mortality was 5\%. Global HF rate was 517 cases per 100.000 elderly per year (270 cases/100.000 elderly males and 695/100.000 elderly females). In-hospital mortality was hig her in males than females $(8,1 \%$ vs $4,2 \%$, respectively), increases pro gressively with age and it is higher during winter months. Factors asso ciated with mortality were old age, male gender, fractured in winter, and living in Regions others than Mediterranean and Canary Islands.

Conclusions: Hip fracture is a condition that occurs more commonly in elderly people. It is more frequent in females and shows a great varia tion in incidence, mortality and length of hospital stay through different Spanish regions.

KEY WORDS: Hip fracture. Elderly. Epidemiology.

Serra JA, Garrido G, Vidán M, Marañón E, Brañas F, Ortiz J. Epidemiología de la fractura de cadera en ancianos en España. An Med Inter na (Madrid) 2002; 19: 389-395.

\section{INTRODUCCIÓN}

La fractura de la extremidad proximal del fémur o fractura de cadera (FC) es la complicación más importante de la osteoporosis en términos de mortalidad, morbilidad y costes (1). Puede producirse a cualquier edad pero aproximadamente el $90 \%$ de los casos ocurren en personas de más de 64 años (2) y teniendo en cuenta las tendencias demográficas es de prever un aumento del número de fracturas de cadera en los próximos años (3). Existen multitud de trabajos que analizan la incidencia de la FC tanto en España (4-8) como en el resto de Europa (9-11), Estados Unidos de América (12), y Asia (13). Sin embargo la mayoría de estos trabajos analizan la incidencia en relación al total de la población o al grupo de mayores

Trabajo aceptado: 5 de mayo de 2002

Correspondencia: José Antonio Serra Rexach. Servicio de Geriatría. Hospital General Universitario Gregorio Marañón. C/ Doctor Esquerdo, 46. 28007 Madrid. 
de 49 años, sin hacer referencia específica al grupo de mayores de 64 años. Pensamos que podría ser más interesante referir los datos a la población anciana ya que además de ser un colectivo con unas características específicas, es el grupo de edad dónde se producen la mayor parte de las FC. Por ello nos proponemos analizar la epidemiología de la FC en la población anciana tanto a nivel nacional así como las variaciones entre las distintas Comunidades Autónomas.

\section{SUJETOS Y MÉTODOS}

Se ha diseñado un estudio longitudinal de seguimiento analizando los datos de incidencia de FC (identificada por el código 820.0 al 820.9 de la Clasificación Internacional de Enfermedades) durante los años 1996, 1997, 1998 y 1999, obtenidos a partir del registro nacional del Conjunto Mínimo Básico de Datos del Ministerio de Sanidad y Consumo. Las variables analizadas han sido: edad, sexo, fecha de ingreso, fecha de alta, mortalidad hospitalaria y Comunidad Autónoma. Los datos de la población española para los diferentes años se han obtenido del Instituto Nacional de Estadística (INE) a partir de proyecciones realizadas sobre el ultimo censo disponible (año 1991).

Análisis estadístico. Se han calculado las tasas de fractura de cadera mediante tasa o densidad de incidencia bruta, de tal forma que en el numerador se han recogido todas las fracturas aparecidas en los 4 años estudiados y en el denominador la suma de todos los individuos en riesgo (esto es de las distintas poblaciones para cada año) (14). Además se ha calculado la tasa de incidencia ajustada por grupos etarios y por sexo para conocer el peso real de la edad y el sexo en la aparición de la fractura de cadera. Para este ajuste se consideró como población estándar la población española a 1 de julio de 1999. Las variables cualitativas se han expresado mediante porcentajes y tablas de frecuencia y las variables cuantitativas mediante la media y su desviación estándar. Por último, en el análisis de la mortalidad hospitalaria de los pacientes con fractura de cadera se ha realizado un análisis bruto y posteriormente un análisis ajustado mediante una regresión logística buscando un modelo explicativo mediante el método enter utilizando el programa SPSS v10.

\section{RESULTADOS}

Datos generales. Desde el 1 de enero de 1996 al 31 de diciembre de 1999 se registraron 147.066 casos de FC en España. En 683 casos faltaba alguna de las variables a analizar por lo que decidimos excluirlas del estudio. Por lo tanto el total de casos incluidos fueron 146.383 de los cuales 130.414 correspondían a pacientes iguales o mayores de 65 años. En la tabla I aparecen recogidas las variables referidas a población anciana y por comunidades autónomas. Cerca del $90 \%$ de todos los casos de FC se producen en ancianos, siendo el $78 \%$ mujeres. La estancia media hospitalaria varía desde los 12-13 días de Baleares, Castilla la Mancha, Comunidad Valenciana, La Rioja y Ceuta, hasta los 23-24 días de Canarias y Melilla. La edad media está prácticamente en todos las comunidades por encima de los 80 años . La mortalidad hospitalaria global es del $5 \%$ pero existe también una gran variabilidad entre Comunidades: $2-3 \%$ en la Comunidad Valenciana y Navarra o 7-8\% en Aragón, Galicia y Ceuta.

Incidencia bruta y estratificada por edad y sexo. En España la incidencia global de FC en ancianos es de 517 casos por 100.000 habitantes y año; 270 casos por 100.000 varones ancianos y año y 695 por 100.000 mujeres ancianas y año (Tabla II).

\section{TABLA I}

\section{DATO S GENERALES (1996-1999)}

\begin{tabular}{|c|c|c|c|c|c|}
\hline $\begin{array}{l}\text { Comunidad Autómoma } \\
\text { (Número total de personas } \\
\text { con fractura de cadera) }\end{array}$ & Mayor de 64 años & M ujeres & Estancia (días) & Edad (años) & M ortalidad \\
\hline Andalucía (24.142) & $20.995(87)$ & 77,8 & $14,88 \pm 12,28$ & $80,95 \pm 7,27$ & $727(3,5)$ \\
\hline Aragón (6.020) & $5.541(92)$ & 78,9 & $17,24 \pm 12,21$ & $82,78 \pm 7,32$ & $459(8,3)$ \\
\hline Principado de Asturias (4.289) & $3.823(89,1)$ & 79,1 & $18,87 \pm 13,73$ & $82,27 \pm 7,45$ & $256(6,7)$ \\
\hline Islas Baleares (2.618) & $2.289(87,4)$ & 75,8 & $12,48 \pm 10,06$ & $81,72 \pm 7,45$ & $127(5,5)$ \\
\hline Canarias (1.993) & $1.630(81,8)$ & 74,0 & $23,06 \pm 24,85$ & $81,09 \pm 7,77$ & $74(4,5)$ \\
\hline Cantabria (2.041) & $1.825(89,4)$ & 80,4 & $14,69 \pm 14,91$ & $82,47 \pm 7,57$ & $99(5,4)$ \\
\hline Castilla La M ancha (7.786) & $7.049(90,5)$ & 76,6 & $13,33 \pm 10,14$ & $81,81 \pm 7,29$ & $383(5,4)$ \\
\hline Castilla y León (9.589) & $8.710(90,8)$ & 77,3 & $16,20 \pm 10,67$ & $82,91 \pm 7,58$ & $487(5,6)$ \\
\hline Cataluña (30.026) & $27.043(90,1)$ & 77,1 & $15,58 \pm 11,79$ & $82,32 \pm 7,33$ & $1,268(4,7)$ \\
\hline Comunidad Valenciana (14.714) & $13.107(88,7)$ & 77,0 & $13,06 \pm 8,59$ & $81,30 \pm 7,24$ & $292(2,2)$ \\
\hline Extremadura (3.811) & $3.443(90,3)$ & 79,4 & $17,55 \pm 10,55$ & $81,80 \pm 7,48$ & $155(4,5)$ \\
\hline Galicia (8.999) & $7.860(87,3)$ & 79,4 & $19,21 \pm 15,61$ & $82,55 \pm 7,44$ & $582(7,4)$ \\
\hline Comunidad de M adrid (16.127) & $14.500(89,9)$ & 79,8 & $19,18 \pm 19,03$ & $82,99 \pm 7,40$ & $938(6,5)$ \\
\hline M urcia (3.271) & $2.814(86)$ & 76,9 & $14,36 \pm 8,48$ & $80,92 \pm 7,46$ & $167(5,9)$ \\
\hline Comunidad de Navarra (2.475) & $2.237(90,4)$ & 82,0 & $15,35 \pm 8,42$ & $82,88 \pm 7,10$ & $73(3,3)$ \\
\hline País Vasco (6.849) & $6.104(89,1)$ & 79,3 & $16,54 \pm 9,25$ & $82,62 \pm 7,36$ & $392(6,4)$ \\
\hline La Rioja (1.258) & $1.145(91)$ & 80,0 & $13,49 \pm 9,07$ & $82,99 \pm 7,30$ & $61(5,3)$ \\
\hline Ceuta (208) & $160(76,9)$ & 74,4 & $12,37 \pm 10,08$ & $79,90 \pm 8,07$ & $12(7,5)$ \\
\hline M elilla (167) & $139(83,2)$ & 68,3 & $24,5 \pm 19,52$ & $80,09 \pm 7,71$ & $6(4,3)$ \\
\hline TOTAL (146.383) & $130.414(89,1)$ & 78 & $15,99 \pm 12,98$ & $82,07 \pm 7,39$ & $6570(5,0)$ \\
\hline
\end{tabular}


TABLA II

\begin{tabular}{|c|c|c|c|c|c|c|}
\hline & \multicolumn{2}{|c|}{ VARONES } & \multicolumn{2}{|c|}{ M UJERES } & \multicolumn{2}{|c|}{ AM BO S SEXO S } \\
\hline & TI* bruta & TI ajustada & TI bruta & TI ajustada & TI bruta & TI ajustada \\
\hline Andalucia & 282 & 413 & 709 & 983 & 531 & 754 \\
\hline Aragón & 275 & 351 & 793 & 1039 & 568 & 743 \\
\hline Asturias & 230 & 333 & 602 & 805 & 450 & 619 \\
\hline Baleares & 292 & 382 & 529 & 689 & 430 & 562 \\
\hline Canarias & 136 & 182 & 288 & 387 & 221 & 301 \\
\hline Cantabria & 230 & 294 & 657 & 839 & 482 & 623 \\
\hline Castillla La M ancha & 290 & 378 & 760 & 1011 & 552 & 734 \\
\hline Castilla y León & 220 & 265 & 569 & 709 & 418 & 518 \\
\hline Cataluña & 363 & 521 & 868 & 1.150 & 658 & 897 \\
\hline Comunidad Valenciana & 290 & 406 & 704 & 975 & 530 & 741 \\
\hline Extremadura & 223 & 304 & 633 & 811 & 460 & 604 \\
\hline Galicia & 194 & 246 & 511 & 644 & 382 & 482 \\
\hline Comunidad de $\mathrm{M}$ adrid & 250 & 381 & 652 & 875 & 492 & 685 \\
\hline Murcia & 252 & 384 & 623 & 881 & 464 & 677 \\
\hline Comunidad de Navarra & 254 & 334 & 858 & 1079 & 601 & 773 \\
\hline País Vasco & 228 & 363 & 618 & 845 & 456 & 659 \\
\hline La Rioja & 271 & 357 & 816 & 1114 & 582 & 796 \\
\hline Ceuta y M elilla & 322 & 540 & 582 & 752 & 473 & 657 \\
\hline Total Nacional & 270 & 373 & 695 & 929 & 517 & 703 \\
\hline
\end{tabular}

* Tasa de incidencia/ 100.000 personas/ año.

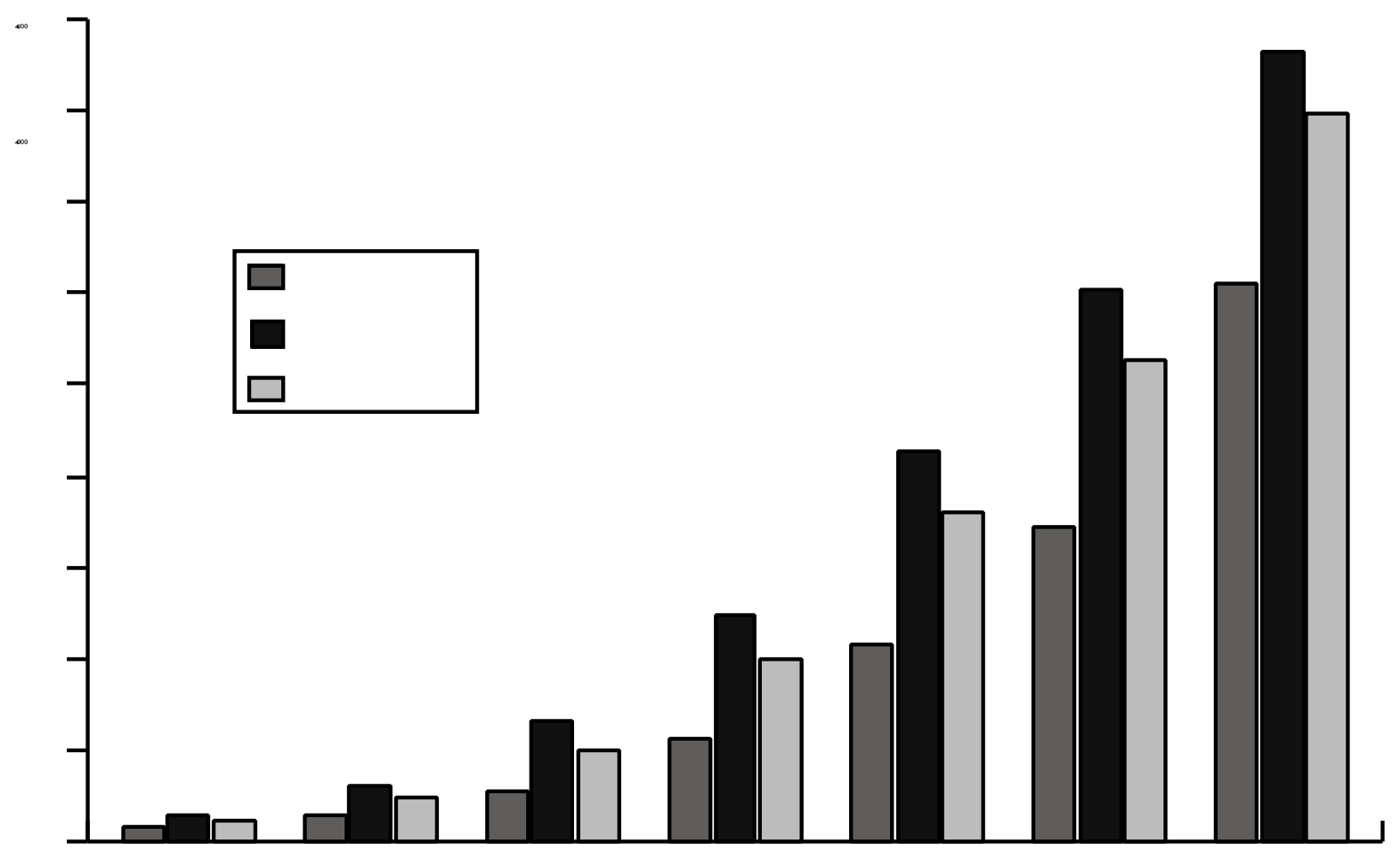

Fig. 1. Incidencia de fractura de cadera en España por edades. 
Existe una gran diferencia en la incidencia entre Comunidades, siendo la más baja en Canarias y la más alta en Cataluña (221 y 658 fracturas de cadera por 100.000 ancianos y año respectivamente). Por grupos de edad la incidencia va aumentando exponencialmente desde los 107 casos por 100.000 ancianos y año entre los 65 y los 69 años hasta alcanzar los 3992 casos por 100.000 ancianos y año para los mayores de 94 años (Fig. 1). La incidencia en mujeres es prácticamente el doble que en los varones en todos los tramos de edad hasta los 94 años (Fig. 1).

Tasas ajustadas por edad y sexo. Las variaciones en la incidencia encontradas en las distintas comunidades autónomas podría deberse a diferencias en la estructura de la población de cada Comunidad. Para poder comparar mejor la incidencia entre ellas es preciso normalizarlas, y para ello ajustamos las tasa de incidencia específica respecto a la población de España, es decir el número de fracturas por 100.000 habitantes y año que cabría esperar en cada CCAA si su distribución, en lo que respecta a edad y sexo, fuera la de la población estándar. Como se aprecia en la tabla II las diferencias entre las CCAA son similares a las encontradas analizando las tasas de incidencia bruta. Estos datos sugieren que las diferencias no se deben a una distinta composición en la población en las CCAA en lo que respecta a sexo y edad, sino a otros factores.

Variación estacional. Como se aprecia en la figura 2 el mayor porcentaje de las FC en ancianos se producen en el invierno $(26,8 \%)$ y el menor en verano $(23,4 \%)$. No obstante existe una gran variabilidad entre las CCAA. La época de menor incidencia es el otoño en Cantabria, Castilla León, Ceuta y Melilla y la primavera en Castilla la Mancha, Extremadura y Murcia. En las comunidades de Aragón y Navarra no existe prácticamente ninguna variación en la incidencia de FC a lo largo de las distintas estaciones.

Mortalidad hospitalaria. La mortalidad hospitalaria global de ancianos con FC durante los 4 años del estudio ha sido del $5 \%$, siendo casi el doble en los varones que en las mujeres ( 8,1 y 4,2 respectivamente). Como se aprecia en la figura 3 , la mortalidad aumenta con la edad en ambos sexos: En mujeres desde un $1,7 \%$ entre 65 a 69 años hasta un $10,9 \%$ en las mayores de 99 años y en varones desde 3,6\% en los de 65 a 69 años hasta un 16,9\% en los mayores de 99 años.

En la figura 4 se muestra la variación de la mortalidad hospitalaria en función de la estación del año en la que se produce el ingreso hospitalario. Como se puede observar la mortalidad en verano es más baja que en el invierno (4,6 y 5,6\% respectivamente). En algunas comunidades la época de menor mortalidad es la primavera (Castilla y León, Extremadura y Madrid) y en otras el otoño (Baleares y Castilla La Mancha). Los datos correspondientes a Ceuta y Melilla no son valorables por el escaso número de pacientes. En las Comunidades de Valencia y Navarra no existe prácticamente variación en la mortalidad entre las distintas estaciones.

Factores de riesgo asociados a mortalidad. Con el fin de analizar qué variables tienen relación con la mortalidad hospitalaria realizamos un análisis bi y multivariante en el que incluimos las variables significativas (Tabla III). Con el fin de comprobar si existía alguna relación entre la zona geográfica y la mortalidad decidimos agrupar las zonas marítimas y de clima cálido (Cataluña, Valencia, Murcia, Andalucía, Baleares y Canarias) y compararlas con el resto de España. Como se puede ver en la tabla III los más ancianos, los varones, los que se fracturan en invierno y los que no habitan en las comu-

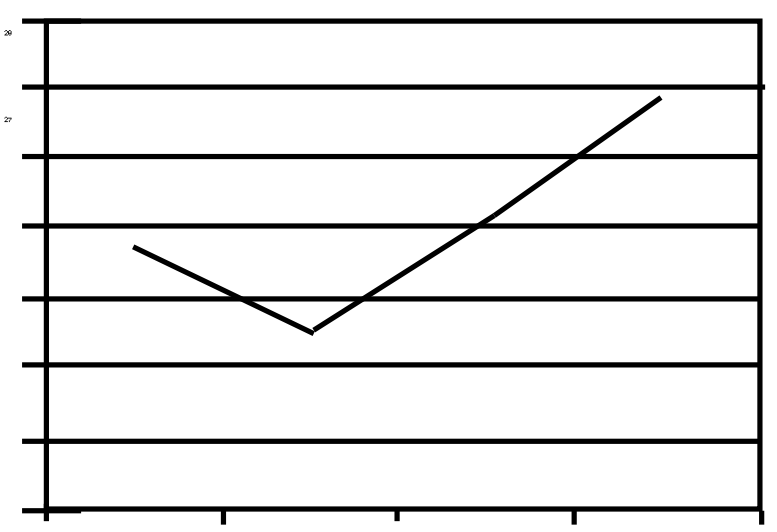

\begin{tabular}{lcccc} 
& Primavera & Verano & Otoño & Invierno \\
\hline Andalucía & 24,7 & 22,6 & 25,1 & 27,6 \\
Aragón & 25,7 & 24,3 & 25,0 & 24,9 \\
Asturias & 24,4 & 22,8 & 25,5 & 27,3 \\
Baleares & 27,1 & 23,6 & 22,1 & 27,3 \\
Canarias & 25,4 & 21,2 & 22,5 & 30,9 \\
Cantabria & 26,5 & 24,9 & 23,3 & 25,3 \\
Castilla La Mancha & 23,6 & 23,9 & 26,6 & 25,9 \\
Castilla y León & 24,4 & 25,9 & 23,9 & 25,8 \\
Cataluña & 25,0 & 22,8 & 25,6 & 26,6 \\
Valencia & 24,2 & 22,9 & 24,7 & 28,2 \\
Extremadura & 23,8 & 26,5 & 23,8 & 26,0 \\
Galicia & 24,2 & 23,8 & 24,6 & 27,3 \\
Madrid & 25,2 & 22,8 & 26,1 & 25,9 \\
M urcia & 22,9 & 23,8 & 23,9 & 29,4 \\
Navarra & 25,0 & 25,0 & 25,1 & 24,9 \\
País Vasco & 24,1 & 23,1 & 25,2 & 27,6 \\
La Rioja & 25,4 & 23,6 & 25,4 & 25,6 \\
Ceuta & 26,9 & 25,6 & 20,6 & 26,9 \\
M elilla & 23,7 & 25,2 & 25,4 & 26,6 \\
\hline
\end{tabular}

Fig. 2. Incidencia de fracturas de cadera. Variación estacional en España.

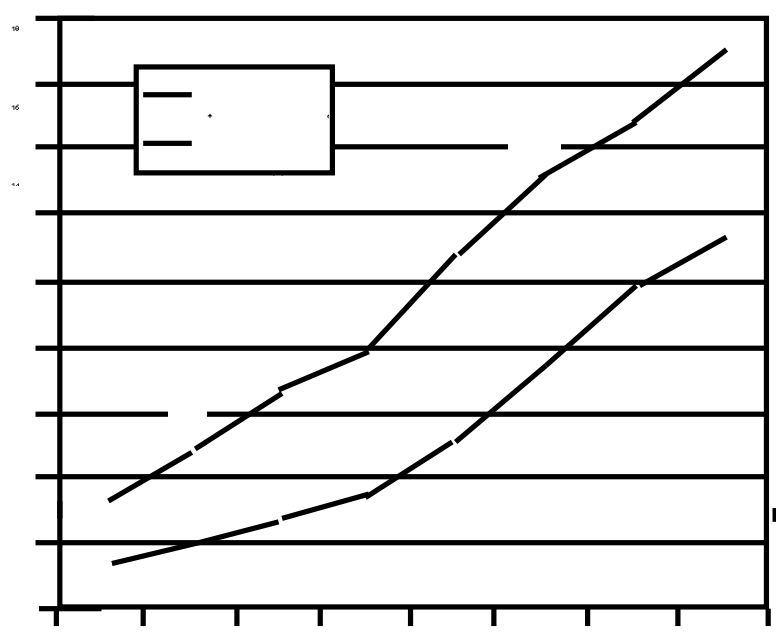

Fig. 3. M ortalidad hospitalaria por grupos de edad y sexo. 


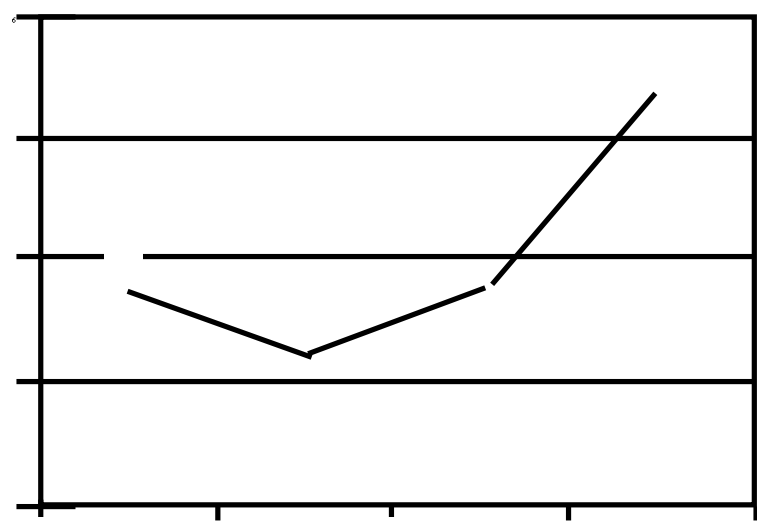

\begin{tabular}{lcccc} 
& Primavera & Verano & Otoño & Invierno \\
\hline Andalucía & 3,3 & 3,0 & 3,2 & 4,2 \\
Aragón & 8,8 & 7,6 & 7,6 & 9,0 \\
Asturias & 7,3 & 5,1 & 6,6 & 7,7 \\
Baleares & 5,6 & 5,6 & 4,8 & 6,1 \\
Canarias & 4,1 & 4,0 & 4,6 & 5,2 \\
Cantabria & 4,3 & 2,9 & 6,6 & 8,0 \\
Castilla La M ancha & 5,2 & 5,5 & 5,0 & 6,1 \\
Castilla y León & 4,5 & 5,1 & 5,6 & 7,1 \\
Cataluña & 4,5 & 4,5 & 5,1 & 4,9 \\
Valencia & 2,0 & 2,0 & 2,7 & 2,2 \\
Extremadura & 3,5 & 4,2 & 4,3 & 5,9 \\
Galicia & 7,4 & 6,6 & 7,5 & 8,1 \\
M adrid & 5,7 & 6,7 & 5,9 & 7,6 \\
M urcia & 6,1 & 5,2 & 5,5 & 6,8 \\
Navarra & 2,7 & 3,4 & 3,6 & 3,4 \\
País Vasco & 7,8 & 5,3 & 5,6 & 6,9 \\
La Rioja & 4,5 & 6,3 & 5,2 & 5,5 \\
Ceuta & 7,0 & 0,0 & 9,1 & 14,0 \\
M elilla & 0,0 & 5,7 & 8,8 & 2,7 \\
\hline
\end{tabular}

Fig. 4. M ortalidad hospitalaria. Variación estacional en España.

nidades del Mediterráneo y Canarias tienen más probabilidades de fallecer en el hospital tras una fractura de cadera tanto en análisis univariante como multivariante.

\section{DISCUSIÓN}

Este estudio muestra que en España cada año aproximadamente 33.000 ancianos, de 82 años de edad media, se fracturan la cadera. El predominio del sexo femenino (tres de cada cuatro casos) y el aumento prácticamente exponencial, de la incidencia por grupos de edad está ampliamente descrito en la literatura (1-13). Este aumento por grupos de edad se observa también analizando separadamente cada una de las CCAA (Datos no mostrados).

Existe una variación de la incidencia por zonas geográficas, hecho descrito ampliamente en la literatura $(10,15,16)$. En nuestro país la incidencia en Canarias (tanto en varones como en mujeres) es menos de la mitad que en el resto de España. No existe una explicación convincente para este hallazgo. No debe tratarse de un problema climatología ya que otras Comunidades igualmente soleadas tienen unas incidencias mucho mayores como Cataluña, Baleares o Andalucía. Como queda demostrado en la tabla II, estas diferencias no se deben a una distinta composición en la población de las CCAA respecto a edad y sexo. Tampoco puede explicarse por una baja fiabilidad de los datos obtenidos del registro del Ministerio de Sanidad ya que en el trabajo prospectivo de Sosa y cols. (7) en el que visitaban en persona todos los hospitales públicos y privados de Gran Canaria para recoger datos de pacientes con $\mathrm{FC}$, los resultados eran muy similares: 160 casos por 100.000 varones mayores de 60 años (136 casos por 100.000 mayores de 65 años en nuestro estudio) y 376 casos por 100.000 mujeres ( 288 casos en nuestro estudio). Podría deberse a unos niveles anormalmente bajos de vitamina D descritos en población anciana española (17-19) aunque no hemos encontrado trabajos que analicen los niveles de esta vitamina en ancianos canarios. También podría deberse a diferencias en el nivel económico, ya que se ha descrito los bajos ingresos económicos como un factor de riesgo de $\mathrm{FC}$, o en el grado de actividad física (20). Es altamente probable que los factores genéticos jueguen un importante papel en esta baja incidencia.

A nivel nacional, el mayor porcentaje de FC se produce en el invierno y el menor en verano, pero no encontramos una razón convincente para explicar este hecho. Se ha sugerido que el clima frío podría influir al facilitar la posibilidad de caída (21). Sin embargo en nuestro estudio parece poco probable al ver los datos de la Comunidad Canaria (con una mínima variación de la temperatura a lo largo del año) en la que el $30 \%$ de las FC se producen en invierno y el $21 \%$ en verano. Por el contrario, en las Comunidades de Aragón y Navarra (con una gran diferencia de la temperatura entre estaciones) no existe prácticamente variación alguna en la incidencia de FC a lo largo del año. Otros estudios sugieren que no existe variación estacional en la incidencia de FC (22) ni asociación entre los cambios de las condiciones ambientales y el riesgo de FC (23). Además, la mayoría de las FC ocurren en el interior de los domicilios, lo que explicaría la ausencia de variación estacional.

Analizando la estancia hospitalaria se observa una gran variabilidad entre 12 y 24 días. Este hecho puede deberse a múltiples factores (24-26): la existencia de protocolos de actuación específicos; la espera preoperatoria; la disponibilidad de servicios de rehabilitación y/o de centros de convalecencia; la colaboración con otras especialidades que pueda disminuir la aparición y el número de complicaciones etc.

En España la mortalidad hospitalaria de ancianos con fractura de cadera es del $5 \%$, variando entre un 2-3\% hasta un 7-8\%. En la literatura se pueden recoger variaciones entre el $1,5 \mathrm{y} \mathrm{el}$ $16 \%$ (27). Varios son los factores relacionados con la mortalidad tras FC: la edad, el sexo masculino, las co-morbilidades (especialmente la demencia), la situación funcional previa, la institucionalización y las complicaciones perioperatorias. En algunos estudios al igualar por otras variables la edad no es predictora de mortalidad. Así, un estudio reciente realizado en nuestro país que analiza los predictores de mortalidad mediante regresión logística múltiple, demuestra que el sexo masculino, la demencia y la mala situación funcional previa a la FC, pero no la edad, son marcadores de mal pronóstico (28). En nuestro estudio los varones tienen una mortalidad mayor que las mujeres. Sin embargo la relación entre el sexo y la mortalidad también es controvertida aunque la mayoría de los autores no encuentran diferencias después de controlar otros factores de riesgo (29, 
TABLA III

FACTO RES DE RIESGO ASO CIADO S A LA M ORTALIDAD HOSPITALARIA EN ANCIANOS CON FRACTURA DE CADERA

\begin{tabular}{|c|c|c|c|c|c|c|}
\hline Variable & $\begin{array}{l}\text { OR* } \\
\text { bruta }\end{array}$ & IC $95 \% \#$ & $p$ & $\begin{array}{c}\text { OR* } \\
\text { ajustada }\end{array}$ & IC $95 \% \#$ & $p$ \\
\hline
\end{tabular}

Edad (años)

(sobre 65-69)

70-74

75-79

1,23

1,04-1,46

1,60

80-84

1,99

85-89

2,85

1,37-1,87

0,014

1,27

0,000

1,71

1,71-2,31

0,000

2,17

2,45-3,30

0,000

3,07

90-94

4,01

3,45-4,67

0,000

4,30

$4,37-6,21 \quad 0,000 \quad 5,52$

95-99

5,21

$4,02-9,07$

0,000

6,29

1,07-1,51

0,049

$1,46-2,00 \quad 0,000$

$1,86-2,52 \quad 0,000$

2,65-3,56 $\quad 0,000$

$3,69-5,00 \quad 0,000$

4,62-6,59 $\quad 0,000$

\section{Sexo}

(sobre mujer)

Hombre

2,01

$1,91-2,12$

0,000

2,20

$2,08-2,32$

0,000

Estación
(sobre verano)
Primavera
O toño
Invierno

$\mathrm{CCAA}^{\&}$

(Sobre resto)

Cataluña, Valencia, M urcia,

Andalucía, Baleares y Canarias

1,03
1,06
1,22

0,95-1,11

$0,99-1,14$

0,405

1,03

1,14-1,31

0,081

1,06

0,95-1,11

0,385

0,000

1,23

$0,98-1,14$

0,094

$1,15-1,32$

0,000

* OR $=$ Odds ratio

\#IC = Intervalo de confianza

${ }^{\star}$ CCAA $=$ Comunidad Autónoma

30). En nuestro estudio, los factores de riesgo relacionados con la mortalidad son la edad, el sexo masculino, sufrir la fractura en invierno y en determinadas áreas geográficas. Estos datos deben tomarse con cautela ya que no hemos podido controlar los principales factores como son las enfermedades acompañantes, las complicaciones perioperatorias y la situación funcional previa a la fractura.

No encontramos en la literatura datos que analicen la variación estacional de la mortalidad en ancianos con FC. Es probable que durante los meses de invierno las posibilidades de sufrir complicaciones, que pueden derivar en muerte, son mayores como ocurre en otras patologías.

El principal interés de este estudio radica en que es el primer trabajo que describe a nivel nacional la incidencia de fractura de cadera en ancianos. Estos datos pueden ser de utilidad para planificar recursos sanitarios destinados a esta patología (número de ingresos previstos, porcentaje de ocupación de camas en los Servicios de Cirugía Ortopédica y Traumatología, necesidades de camas de rehabilitación y convalecencia, necesidades de colaboración con otras especialidades como geriatría, medicina interna o rehabilitación, etc). También pueden utilizarse estos datos para comparar nuestros resultados con los obtenidos en otros Centros de nuestro entorno.

La fractura de cadera es una de las fracturas más fáciles de cuantificar ya que prácticamente siempre requiere de ingreso hospitalario lo que facilita su registro. Sin embargo una posible limitación de este trabajo es la fiabilidad de los registros a nivel nacional. Pero al comparar los datos con los obtenidos registrando todos los ingresos por hospitales podemos comprobar como los resultados son muy parecidos. Ya hemos comentado los datos del trabajo de Sosa y cols. en Gran Canaria (7). La incidencia por edad y sexo y la variación estacional también fueron analizadas por Altadill y cols. quienes revisaron las historias clínicas de todos los pacientes ingresados con el diagnóstico de fractura de cadera en los hospitales públicos y privados de dos áreas sanitarias de Asturias, llegando a resultados similares a los nuestros (5). Los datos descritos por Olmos y cols. revisando las historias de todos los pacientes con FC atendidos en Cantabria durante 1 año (4) son también similares a los descritos por nosotros.

En conclusión este estudio demuestra la elevada incidencia de fractura de cadera en ancianos españoles, con una gran variabilidad en lo que respecta a edad, sexo, estancia, mortalidad hospitalaria e incidencia estacional. Son necesarios estudios encaminados a optimizar el tratamiento de esta patología tanto en lo que respecta al tratamiento hospitalario como a conseguir una mayor recuperación de la situación funcional.

\section{AGRADECIMIENTOS}

Este trabajo ha sido en parte financiado por la Beca del Fondo de Investigación Sanitaria 99/0342 a los Dres. Serra, Vidán y Ortiz. 


\section{Bibliografía}

1. Youm T, Koval KJ, Zuckerman JD. The economic impact of geriatric hip fractures. Am J Orthop. 1999; 28: 423-8

2. Gillespie WJ. Hip fracture. BMJ 2001; 322: 968-975

3. Kannus P, Niemi S, Parkkari J, Palvanen M, Vuori Y, Järvinen M. Hip fractures in Finland between 1970 and 1997 and predictions for the future. Lancet 1999; 353: 802-805

4. Olmos JM, Martínez J, García J, Matorras P, Moreno JJ, GonzálezMacías J. Incidencia de la fractura de cadera en Cantabría. Med Clin (Barc) 1992; 99: 729-731

5. Altadill A, Gómez-Alonso C, Virgós MJ, Díaz-López B, Cannata JB. Epidemiología de la fractura de cadera en Asturias. Med Clin (Barc) 1995; 105: 281-286

6. Arboleya LR, Castro MA, Bartolomé E, Gervás L, Vega R. Epidemiología de la fractura de cadera en la provincia de Palencia. Rev Clin Esp 1997; 197: 611-617

7. Sosa M, Segarra MC, Hernández D, González A, Limiñana JM, Betancor P. Epidemiology of proximal femoral fracture in Gran Canaria (Canary Islands). Age Ageing 1993, 22: 285-288

8. Lizaur A, Motoza JM, Gutiérrez P. Incidencia específica por edad y sexo de las fracturas proximales del fémur. Rev Ortop Traum 1989; 33: 300-304

9. Kamis JA. The incidence of hip fracture in Europe. Osteoporos Int 1993 (Suppl 1): S10-15

10. Elffors I, Allander E, Kanis JA, Gullberg B, Johnell O, Dequeker J et al. The variable incidence of hip fracture in Southern Europe: The MEDOS study. Osteoporos Int 1994; 4: 253-263

11. Lofthus CM, Osnes EK, Falch JA, Kaastad TS, Kristiansen IS, Nordslestten L et al. Epidemiology of hip fracture in Oslo, Norway. Bone 2001; 29: 413-418

12. Hinton RY, Lennox DW, Ebert FR, Jacobsen SJ, Smith GS. Relative rates of fracture of the hip in the United States: Geographic, sex and age variations. J Bone Joint Surg Am 1995; 77: 695-701

13. Lau EMC, Lee JK, Suriwongpaisal P, Saw SM, Das De S, Khir A et al. the incidence of hip fracture in four Asian countries: The Asian Osteoporosis Study (AOS). Osteoporos Int 2001; 12: 239-243

14. Rothman KJ, Greenland S. Measures of Disease Frequency. En K.J. Rothman, S. Greenland, editores. Modern Epidemiology. Filadelfia: Lippincott-Raven Publisher, 1998; 24-46

15. Jacobsen SJ, Goldberg J, Miles TP, Brody JA, Stiers W, Rimm AA. Regional variation in the incidence of hip fracture: US white women aged 65 years and older. JAMA 1990; 264: 500-502

16. Finsen V, Benum $P$, Changing incidence of hip fractures in rural and urban areas of central Norway. Clin Orthop 1987; 218: 104-111

17. Quesada JM, Jans I, Benito P, Jiménez JA, Bouillon R. Vitamin D status of elderly people in Spain. Age Ageing 1989; 18: 392-397

18. González-Clemente JM, Martínez-Osaba MJ, Minarro A, Delgado MP, Mauricio D, Ribera F. Hipovitaminosis D: Elevada prevalencia en ancianos en Barcelona. Factores asociados. Med Clin (Barc) 1999; 113: 641-645

19. Aguado P, del Campo MT, Garces MV, Gonzalez-Casaus ML, Bernad $\mathrm{M}$, Gijon-Baños $\mathrm{J}$ et al. Low vitamin D levels in outpatient postmenopausal women from a rheumatology clinic in Madrid, Spain: their relationship with bone mineral density. Osteoporos Int 2000; 11: 739-744

20. Cummings SR, Nevitt MC, Browner WS, Stone K, Fox KM, Ensrud KE et al . Risks factors for hip fracture in white women. N Eng J Med 1995; 332: 767-773

21. Levy AR, Bensimon DR, Mayo NE, Leighton HG. Inclement weather and the risk of hip fracture. Epidemiology 1998; 9: 172-177

22. Baudoin C, Fardellone P, Potard V, Sebert JL. Fractures of the proxima femur in Picardy, France, in 1987. Osteopor Int 1993; 3: 43-49

23. Jacobsen SJ, Sargent DJ, Atkinson EJ, O'Fallon WM, Melton LJ. Population-based study of the contribution of weather to hip fracture seasonality. Am J Epidemiol 1995; 141: 79-83

24. Gonzalez-Montalvo JI, Alarcón T, Saéz P, Bárcena A, Gotor P, Del Río M. La intervención geriátrica puede mejorar el curso clínico de los ancianos frágiles con fractura de cadera. Med Clin (Barc) 2001; 116: 1-5

25. Morrison RS, Chassin MR, Siu AL. The medical consultant's role in caring patients with hip fracture. Ann Inter Med 1998; 128: 1010-1020

26. Cruz-Jentoft AJ, Serra Rexach JA, Lázaro del Nogal M, Gil Gregorio P Ribera Casado JM. La eficacia de la interconsulta geriátrica en pacientes ancianos ingresados en traumatología. An Med Interna (Madrid) 1994; 11: 273-277

27. Hannan EL, Magazinger J, Wang JJ, Eastwood EA, Silberzweig SB, Gilbert $\mathrm{M}$ et al. Mortality and locomotion 6 months after hospitalitation for hip fracture. JAMA 2001; 285: 2736-2742

28. Pagès E, Cuxart A, Iborra J, Olona M, Bermejo B. Fracturas de cadera en el anciano. Determinantes de mortalidad y capacidad de marcha. Med Clin (Barc) 1998; 110: 687-691

29. Myers AH, Robinson EG, Van Natta ML, Michelson JD, Collins K, Baker SP. Hip fractures among the elderly: factors associated with inhospital mortality. Am J Epidemiol 1991; 134: 1128-1137

30. Aharonoff GB, Koval KJ, Skovron ML, Zuckerman JD. Hip fractures in the elderly: predictors of one year mortality. J Orthop Trauma 1997; 11: $162-165$ 